\title{
BIOINFORMATION
}

Discovery at the interface of physical and biological sciences

\section{Annotation of a hypothetical protein (WP_002969292.1) from Brucella abortus}

\author{
Kanchan Rauthan ${ }^{1 \$}$, Divya Goel ${ }^{1 \$}$, Sudhir Kumar ${ }^{1 *}$
}

1Department of Biotechnology, Hemvati Nandan Garhwal University, Srinagar Garhwal, Uttarakhand-246174, India; Sudhir Kumar Email: sudhir.1685@gmail.com; *Corresponding author; \$Equal contribution.

Received April 1, 2019; Accepted April 17, 2019; Published April 30, 2019

DOI: $10.6026 / 97320630015315$

\section{Abstract:}

Brucellosis is a zoonotic disease caused mainly by the bacteria belonging to the genus Brucella, most common of them is Brucella abortus. Genome sequencing of Brucella was completed in 2005. While majority of the proteins were assigned function, a large number of the peptides remained un-annotated and were referred as 'hypothetical'. These hypothetical proteins may contain crucial information about the biology and pathogenesis of the $B$. abortus. Therefore, it is of interest to annotate one such hypothetical protein as a multiple antibiotic resistance regulator protein, MarR. The physiological parameters, localization and the structural features were predicted for this protein which corroborated as the winged-helix type DNA-binding domain superfamily of transcription factors.

\section{Background:}

Brucellosis is a disease with many names such as malta fever, bang's disease, Gibraltar fever, undulant fever and many more. It has been a major cause of concern with almost 5,00,000 cases annually. Brucellosis is mostly found in domestic animals and it is caused by bacteria of Brucella species. Its transmission through untreated or contaminated milk is most common. Brucellosis is potential bioterror threat. Veterinary and abattoir accidents are another way of transmission. Brucella is a small gram-negative aerobic coccobacillus and a facultative intracellular parasite which includes B. abortus, B. melintensis, B. suis, B. neotomae, B. ovis, B. canis etc [1].

The genome of Brucella contains two chromosomes of 2.1 and 1.16 mbp, respectively. The sequenced $B$. abortus genome is reported to have 3,296 ORFs or genes. Out of these 3296 ORFs, 2,158 are situated on Chromosome I and 1,138 are on Chromosome II. It was also reported by the same group that $B$. abortus genome contains many short ORFs, which are less than 100 aa long [2]. Brucella genome is unique because of the absence of plasmids and temperate phage. No evidence showing natural transfer of genetic material in Brucella has been recorded though very low occurrence of transduction has been shown [3]. Comparative genomics study of $B$. abortus with $B$. melintensis and $B$. suis reveals that it shares more identity with $B$. melintensis than the latter [2]. All the species of the Brucella genus share a conserved gene pool with diversity mainly because of recombinations [3].

A large number of protein sequences in Brucella genome have not been annotated and are deposited as hypothetical proteins. A search in the NCBI database using term 'hypothetical proteins' in Brucella abortus genome yielded about 581 protein sequences. We tried to annotate and assign function to most of them (Unpublished data). One of the sequences that got our interest was a hypothetical protein WP_002969292.1 from Brucella abortus and we decided to further investigate and annotate the sequence. A large number of in silico tools were employed to determine the structural and functional characteristics of the sequence. The hypothetical protein was identified as Multiple Antibiotic Resistance Regulatory (MarR) protein.

\section{Methodology: \\ Sequence retrieval and analysis:}




\section{BIOINFORMATION \\ Discovery at the interface of physical and biological science.}

Amino acid sequence of Hypothetical protein WP_002969292.1 from Brucella abortus (hereafter referred as BaHP) was retrieved from NCBI. Physiological parameters were calculated for this sequence by using protparam from ExPASy server [4]. The putative localization of BaHP was predicted using CELLO [5] and CELL-P LOC 2.0 [6]. The protein sequence was subjected to protein blast using blastp program of NCBI [7] to search for similar sequences.

\section{Secondary structure analysis and fold recognition:}

Secondary structures of BaHP were predicted using SABLE [8] and J PRED server [9]. PHYRE2 [10] server were used for fold recognition analysis and InterProScan [11] was used to search architectural motifs of the BaHP.

\section{Structural modelling and analysis:}

The structural modelling of BaHP was performed in a stepwise procedure from Swiss-Model against the proteins with similar sequence. The search identified crystal structure of Ruegeria pomeroyi (3CJN) MarR protein [12] as the template. The predicted 3$\mathrm{D}$ model was downloaded and refined using MODRefiner [13].

The refined structure model was chosen on the basis of the stereochemistry quality report generated using PROCHECK (used for inspection of $\Psi / \Phi$ Ramachandran plot) in PDBsum [14]. It was also used to find out the residues responsible for dimerization of the BaHP molecule. The DNA binding residues were predicted by aligning the structure of BAHP with structure of RovA master virulence regulator (PDB id 4AIK) with promoter DNA [15].

\section{Phylogenetic analysis:}

The full-length MarR protein sequences from different bacteria were obtained from NCBI and used to perform phylogenetic analysis using MegaX program [16]. The tree was calculated using the Neighbour-Joining method.

Table 1: Physicochemical properties of hypothetical protein WP_002969292.1 calculated using Protparam.

\begin{tabular}{|c|c|c|}
\hline NCBI Accession ID & & WP_002969292.1 \\
\hline No. of Amino acids & & 85 \\
\hline Molecular weight, $\mathrm{Mw}(\mathrm{Da})$ & & 9685.04 \\
\hline Theoretical PI & & 6.60 \\
\hline Extinction coefficient & & 1490 \\
\hline Instability index & Computed & 46.71 \\
\hline & Classification & Unstable \\
\hline Aliphatic index & & 76.94 \\
\hline Grand Average Of Hydropathicity (GRAVY) & & -0.978 \\
\hline
\end{tabular}

ISSN 0973-2063 (online) 0973-8894 (print)

Results:

Sequence analysis of BaHP:

The search for protein sequences using word 'hypothetical' yield a large number protein sequences in the genome of $B$. abortus in NCBI which has not yet been annotated or characterized. One of the hypothetical proteins carrying accession number WP_002969292.1 showed similarity with MarR protein in protein Blast. The protein sequence of this hypothetical protein is:

\section{MVKRASETDQRQSHVYLTQAGLQTIKAIEKSIRKTEKDMLKGLD KKDRKSLLKMLSRMEGNLVLRGAARVADEPETEPQEDDEAE}

\section{Physicochemical properties:}

Protparam tool from Expasy server was used to analyse the hypothetical protein. It predicted the molecular weight of this protein at about $9685.04 \mathrm{Da}$ and reported an unstable protein based on the instability index. The protein was show to be hydrophilic in nature with pI of 6.6 (Table 1) Localization server predicted this protein to be localized predominantly in cytoplasm (Table 2).

Table 2: Prediction of Cellular Localization of the hypothetical protein WP_002969292.1 using different programs

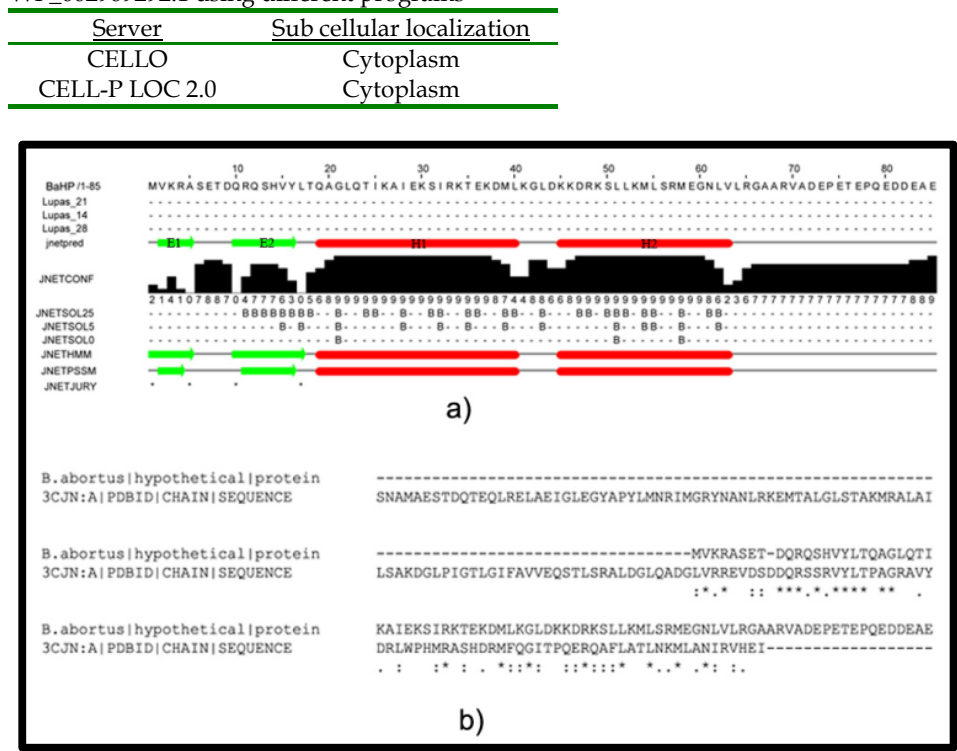

Figure 1: Sequence analysis $\mathrm{BaHP}$ for secondary structure prediction (a) The secondary structures as predicted by JPRED servers. (b) Sequence alignment of BaHP with FASTA sequence of 3CJN (crystal structure of transcriptional regulator, MarR family, from Silicibacter pomeroyi). The BaHP shared about $32 \%$ sequence identity with the 3CJN sequence. 

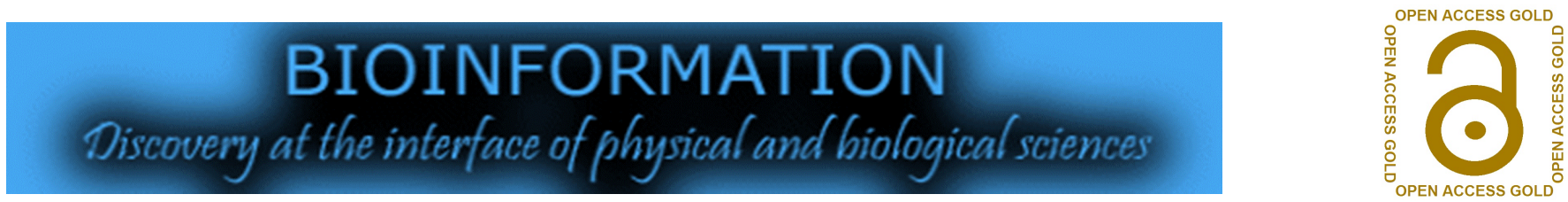

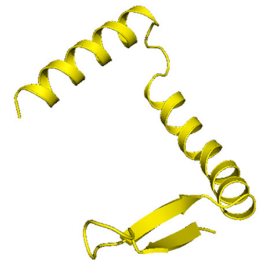

a)

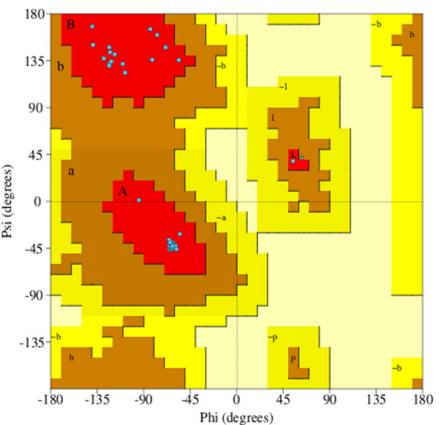

d)

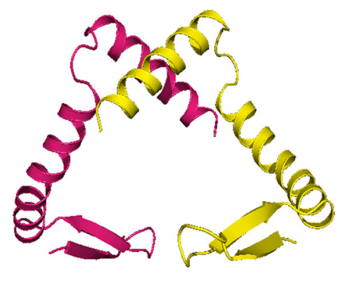

b)

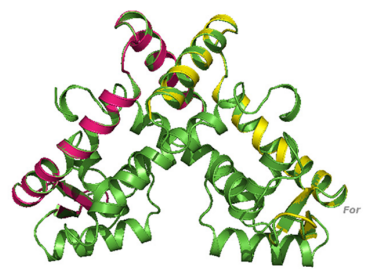

c)

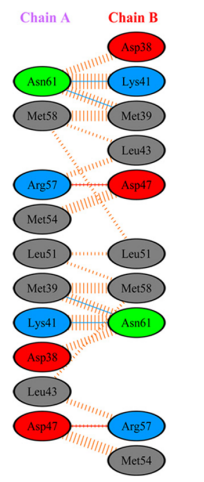

e)

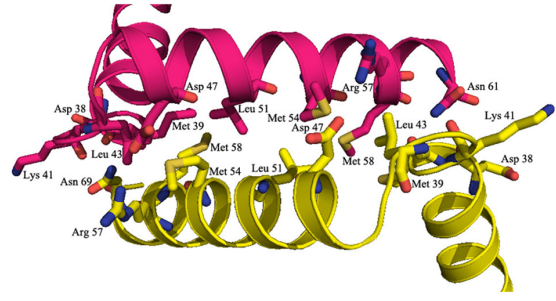

f)

Figure 2: Structural Features of BaHP (a) Model of BaHP showing one monomer containing typical Helix turn Helix (HTH) domain; (b) Dimer of the BaHP model showing interactions between the C-terminal Helix 2 of both monomers; (c) Structural Alignment of BaHP model with its template 3CJN (Green); (d) Ramachandran plot for the predicted model showing 100\% residues in allowed region; 9e) interacting residues at the dimer interface as predicted by PDBsum and (f) zoomed image to show the interactions of different residues in dimer interface

\section{Secondary structure analysis and fold recognition:}

The secondary structures of the BaHP predicted using SABLE and J PRED server predicted 2 small $\beta$-sheets (E1 an E2) and 2 large $\alpha$ helix ( $\mathrm{H} 1$ and $\mathrm{H} 2)$ with little variation in percentage of $\beta$-sheets to be at $7.1 \%$ and that of $\alpha$-helix at $49.4 \%$. Rest of the sequences were in the form of loops. First $\beta$-sheet E1 was predicted to be from Val 2 - Ala 4 (3 aa) and second $\beta$-sheet was from Gln 12 - Leu 17 (6 aa). The $\alpha$-helix H1 ranged between Gln 19- Leu 40 (21 aa) and H2 ranged between Lys 45 - Val 63 (19 aa) (Figure 1a). JPRED tool predicted it to be a member of MarR family transcriptional regulator cluster. Phyre2 predicted the fold as DNA/RNA-binding 3-helical bundle that belong to the "Winged helix" DNA-binding domain superfamily. It also showed that that BaHP belong to the MarR-like transcriptional regulators family. The Phyre2 search predicted that BaHP share a good homology with crystal structure of transcriptional regulator, MarR family, from Silicibacter pomeroyi bearing PDB id 3CJN [12] which was confirmed by sequence alignment (Figure 1b).

Interproscan predicted the BaHP sequence to contain the HelixTurn-Helix (HTH) motif and a HTH marR-type domain between amino acid no. 1 - 60 which belonged to a Winged Helix like DNA binding Domain superfamily. Interproscan result also showed the BaHP to have a DNA-template regulation of transcription biological function and predicted it to be a transcriptional regulator. Combination of these results implies that $\mathrm{BaHP}$ is a transcriptional regulator that belongs to the HTH-MarR type family of winged helix DNA binding protein superfamily. 

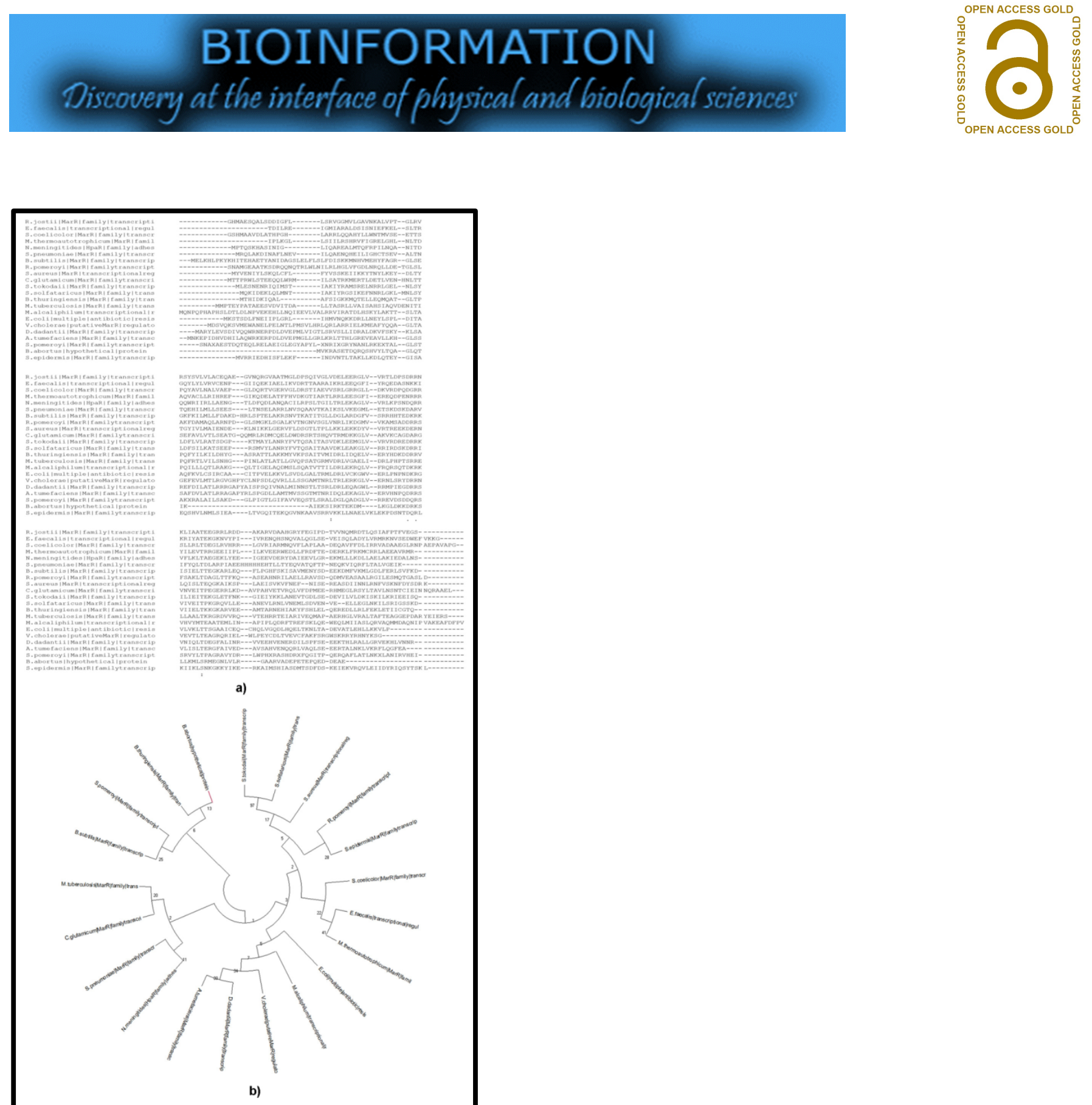

Figure 3: Sequence alignment and phylogenetic analysis. (a) The sequence alignment of MarR protein sequences form different organisms aligned to BaHP using Muscle Program (https://www.ebi.ac.uk/Tools/msa/muscle/). (b) The phylogenetic tree was inferred using the Maximum Parsimony method in MEGA X. The bootstrap consensus tree inferred from 300 replicates is taken to represent the evolutionary history of the taxa analyzed. Branches corresponding to partitions reproduced in less than $50 \%$ bootstrap replicates are collapsed. The MP tree was obtained using the Subtree-Pruning-Regrafting (SPR) algorithm with search level 1 in which the initial trees were obtained by the random addition of sequences (10 replicates). This analysis involved 21 amino acid sequences. There were a total of 190 positions in the final dataset. 


\section{BIOINFORMATION \\ Discovery at the interface of physical and biological sciences}

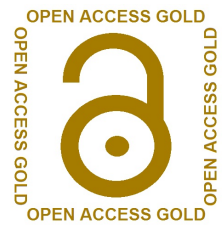

binding affinity of protein [19]. The dimer can also be seen in BaHP structure and can potentially bind to promoter DNA.

\section{Conclusion:}

While the genome sequencing consortiums have lately resulted in influx of large genomic data, majority of protein sequences in these genomes remain un-annotated [20]. These proteins, if investigated systematically can yield a better perspective into the general biology and pathology of the organism. We annotated one such hypothetical protein from Brucella abortus in this study. It was shown to be a member of MarR superfamily of promoter binding proteins using sequence and structural characteristics. The $B$. abortus MarR protein model revealed a highly conserved DNA binding domain (WHT domain) despite having low sequence similarity. Similar kind of approach can be used for annotation of other hypothetical proteins.

\section{References:}

[1] Chritopher Set al. J Lan Physicians 2010 2:5. [PMID: 21346896]

[2] Halling MS et al. J Bac. 2005 187:2715. [PMID: 15805518]

[3] O'Callaghan D \& Whatmore AM, Brief Funct Genomics 2011 10:334. [PMID: 21930657]

[4] http://www.expasy.org/tools/

[5] Yu CS et al. Protein Science 2004 13:1402. [PMID: 16752418]
[6] Chou KC \& Shen HB. Journal of Proteome Research 2006 5:3420. [PMID: 16889410]

[7] https://blast.ncbi.nlm.nih.gov/Blast.cgi

[8] Adamczak R et al. Proteins: Structure, Function and Bioinformatics 2004 56:753. [PMID: 15281128]

[9] Drozdetskiy A, Nucl. Acid Res. 2015 43:W389. [PMID: 25883141]

[10] Kelley LA et al. Nature Protocols 2015 10: 845. [PMID: 25950237]

[11] https://www.ebi.ac.uk/interpro/search/sequence-search

[12] https://www.rcsb.org/structure/3cjn

[13] Xu D \& Zhang Y. Biophysical Journal 2011 101:2525. [PMID: 22098752]

[14] http://www.ebi.ac.uk/thornton-srv/databases/cgibin/pdbsum/

[15] Quade N et al. J Biol. Chem. 2012 287:35796. [PMID: 22936808]

[16] Kumar S et al. Molecular Biology and Evolution 2018 35:1547. [PMID: 29722887]

[17] Grove A, Current Biology 2013 23:R142. [PMID: 23428319

[18] Cohen SP et al. Journal of Bacteriology 1993 175:1484. [PMID: 8383113]

[19] Saridakis V et al. Journal of Molecular Biology 2008 377:655. [PMID: 18272181]

[20] Galperin MY \& Koonin EV, Nucleic Acids Research 2004 32:5452. [PMID: 15479782]

Edited by P Kangueane

Citation: Rauthan et al. Bioinformation 15(5): 315-320 (2019)

License statement: This is an Open Access article which permits unrestricted use, distribution, and reproduction in any medium, provided the original work is properly credited. This is distributed under the terms of the Creative Commons Attribution License
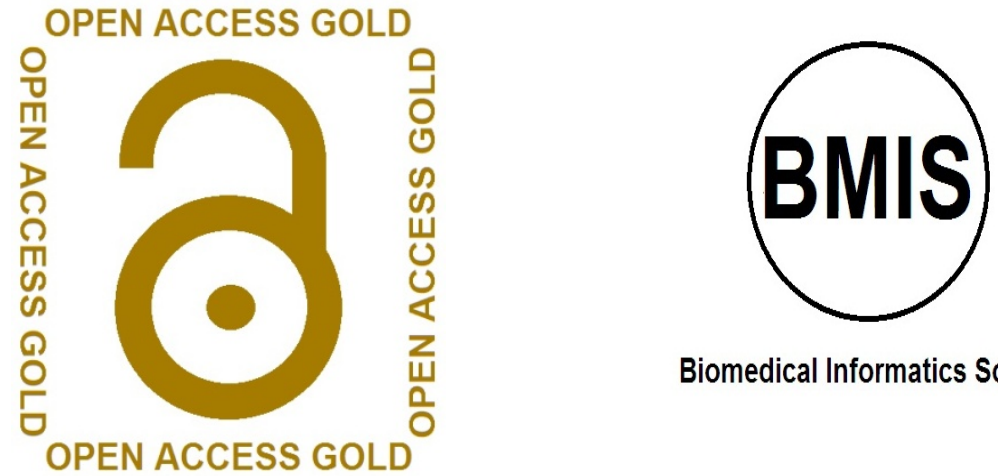

Biomedical Informatics Society

\section{Agro Informatics Society}

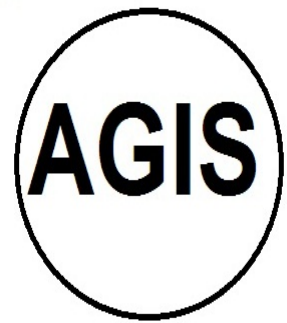

